\title{
Loss of p53 results in protracted electrographic seizures and development of an aggravated epileptic phenotype following status epilepticus
}

\author{
T Engel ${ }^{1}, \mathrm{~K}_{\text {Tanaka }}{ }^{1,2}$, EM Jimenez-Mateos ${ }^{1}$, A Caballero-Caballero ${ }^{1}$, JHM Prehn ${ }^{1}$ and DC Henshall ${ }^{\star, 1}$
}

The p53 tumor suppressor is a multifunctional protein, which regulates cell cycle, differentiation, DNA repair and apoptosis. Experimental seizures up-regulate $\mathrm{p} 53$ in the brain, and acute seizure-induced neuronal death can be reduced by genetic deletion or pharmacologic inhibition of p53. However, few long-term functional consequences of p53 deficiency have been explored. Here, we investigated the development of epilepsy triggered by status epilepticus in wild-type and p53-deficient mice. Analysis of electroencephalogram (EEG) recordings during status epilepticus induced by intra-amygdala kainic acid (KA) showed that seizures lasted significantly longer in p53-deficient mice compared with wild-type animals. Nevertheless, neuronal death in the hippocampal CA3 subfield and the neocortex was significantly reduced at $72 \mathrm{~h}$ in p53-deficient mice. Long-term continuous EEG telemetry recordings after status epilepticus determined that the sum duration of spontaneous seizures was significantly longer in p53-deficient compared with wild-type mice. Hippocampal damage and neuropeptide $Y$ distribution at the end of chronic recordings was found to be similar between p53-deficient and wild-type mice. The present study identifies protracted KA-induced electrographic status as a novel outcome of p53 deficiency and shows that the absence of p53 leads to an exacerbated epileptic phenotype. Accordingly, targeting p53 to protect against status epilepticus or related neurologic insults may be offset by deleterious consequences of reduced p53 function during epileptogenesis or in chronic epilepsy.

Cell Death and Disease (2010) 1, e79; doi:10.1038/cddis.2010.55; published online 7 October 2010

Subject Category: Neuroscience

Epilepsy is a common, chronic neurologic disorder characterized by recurrent seizures. Experimental modeling and human data show that prolonged seizures (status epilepticus) or repeated brief seizures over time can cause neuron loss, cognitive deficits and trigger or exacerbate epilepsy. ${ }^{1,2} \mathrm{~A}$ variety of signaling pathways have been implicated in seizure-induced neuronal death, including those associated with apoptosis. ${ }^{3}$ The tumor suppressor p53 was one of the first such genes implicated in effecting seizure-induced neuronal death. In neurons, p53 is a pleiotropic transcription factor which controls DNA repair, cell cycle progression, differentiation and apoptosis. ${ }^{4}$ Over-expression of p53 induces death of hippocampal neurons, ${ }^{5,6}$ probably via transcriptional induction of the pro-apoptotic Bcl-2 family protein Puma.,8 Excitotoxic insults and prolonged seizures up-regulate p53 in vivo, ${ }^{9,10}$ and p53 levels are also elevated in hippocampus from patients with long-standing temporal lobe epilepsy. ${ }^{11}$ Absence of p53 can prevent glutamate- and kainic acid (KA)-induced death of cortical and hippocampal neurons in vitro. ${ }^{5}$ Moreover, p53-deficient mice are protected acutely against seizure-induced neuronal death in vivo ${ }^{8,12,13}$ and the p53 inhibitor pifithrin- $\alpha$ reduces seizure-induced neuronal death in vitro and in vivo. $8,14,15$
The question of whether neuroprotection has functional antiepileptogenic consequences remains uncertain. ${ }^{1}$ In some studies, a reduction of hippocampal damage after status epilepticus failed to block the later emergence of epilepsy. ${ }^{16,17}$ However, antiepileptogenic effects have been reported in mice protected by epileptic pre-conditioning, ${ }^{18}$ or genetic deletion of puma. ${ }^{8}$ Puma induction after status epilepticus is p53-dependent, ${ }^{8}$ so it is possible that loss of p53 would also confer antiepileptogenic effects in the same model. However, this may be offset by deregulation of p53-controlled functions such as DNA repair, regulation of neurite branching and axonal regeneration, ${ }^{19,20}$ and, possibly, inhibitory neurotransmission. ${ }^{21}$ Interestingly, mice lacking p53 can display neurodegeneration $^{22}$ and worse outcomes after focal cerebral ischemia. $^{23}$

In the present study, we sought to determine the functional significance of p53 in the pathogenesis of epilepsy by characterizing the epileptic phenotype following status epilepticus in mice lacking p53. Our data show that status epilepticus in p53-deficient mice have an altered electroencephalographic (EEG) profile and result in a more severe epileptic phenotype.

\footnotetext{
${ }^{1}$ Department of Physiology \& Medical Physics, Royal College of Surgeons in Ireland, 123 St. Stephen's Green, Dublin 2, Ireland and ${ }^{2}$ Department of Neurosurgery, Mie University School of Medicine, Tsu, Mie, Japan

*Corresponding author: DC Henshall, Department of Physiology \& Medical Physics, Royal College of Surgeons in Ireland, 123 St. Stephen's Green, Dublin 2, Ireland. Tel: + 3531402 8629; Fax: + 3531402 2447; Email: dhenshall@ rcsi.ie

Keywords: apoptosis; Bcl-2; epileptogenesis; hippocampal sclerosis; kainate; video-EEG

Abbreviations: PBS, phosphate-buffered saline; $E E G$, electroencephalogram; $G_{A B A}, \gamma$-amino butyric acid; KA, kainic acid

Received 10.5.10; revised 26.7.10; accepted 12.8.10; Edited by A Finazzi-Agro'
} 


\section{Results}

Normal hippocampal architecture in p53-deficient mice. Before our analysis of their response to status epilepticus, we examined brains from naïve wild-type and p53-deficient mice, as developmental defects in p53deficient mice have been reported. ${ }^{24,25}$ Inspection of coronal brain sections stained for the neuronal nuclear protein NeuN showed normal hippocampal architecture in p53-deficient mice (Figure 1a). Counts of pyramidal neurons from a variety of subfields revealed no differences in cell numbers between wild-type and p53-deficient mice (Figure 1b). Next, we used western blotting to assess levels of the KA receptor GluR6/7 to exclude potential sources of variance in response to KA. Expression of GluR6/ 7 in whole-cell lysates from the amygdala and hippocampus was not different between wild-type and p53-deficient mice (Figure 1c).

Acute hippocampal CA3 and neocortical neuroprotection after status epilepticus in p53-deficient mice. We recently reported that the hippocampal CA3 subfield was less damaged in p53-deficient mice compared with wild-type animals when status epilepticus was induced by intraamygdala $\mathrm{KA}^{8}$ To extend these data, we examined this and other brain regions from wild-type and p53-deficient mice $72 \mathrm{~h}$ after status epilepticus in the same model. As reported, ${ }^{8}$ p53-deficient mice displayed significantly less seizureinduced neuronal death within the ipsilateral CA3 subfield after status epilepticus compared with wild-type animals (Figure $2 a$ and $b$ ). Analysis of the ipsilateral hilar region of the dentate gyrus and the CA1 subfield of the hippocampus in wild-type animals found small and rather variable numbers of degenerating neurons, as previously reported. ${ }^{26}$ There was a trend of neuroprotection in both fields in p53-deficient mice although this did not reach statistical significance (Figure 2c-f). In contrast, seizure-induced neuronal death in the ipsilateral neocortex was also significantly reduced in p53-deficient mice compared with wild-type animals (Figure $2 \mathrm{~g}$ and $\mathrm{h})$.

p53-regulated genes after status epilepticus in p53deficient mice. We recently showed that induction of Puma, a member of the BH3-only subgroup of pro-apoptotic $\mathrm{Bcl}-2$ family proteins, was p53-dependent in the present model and that Puma-deficient mice were protected against seizure-induced neuronal death. ${ }^{8}$ We also established that Noxa, another $\mathrm{BH} 3-$ only protein regulated by $\mathrm{p} 53$, was not induced. ${ }^{8}$ Here, we analyzed hippocampal levels of multidomain pro-apoptotic Bax and the BH3-only protein
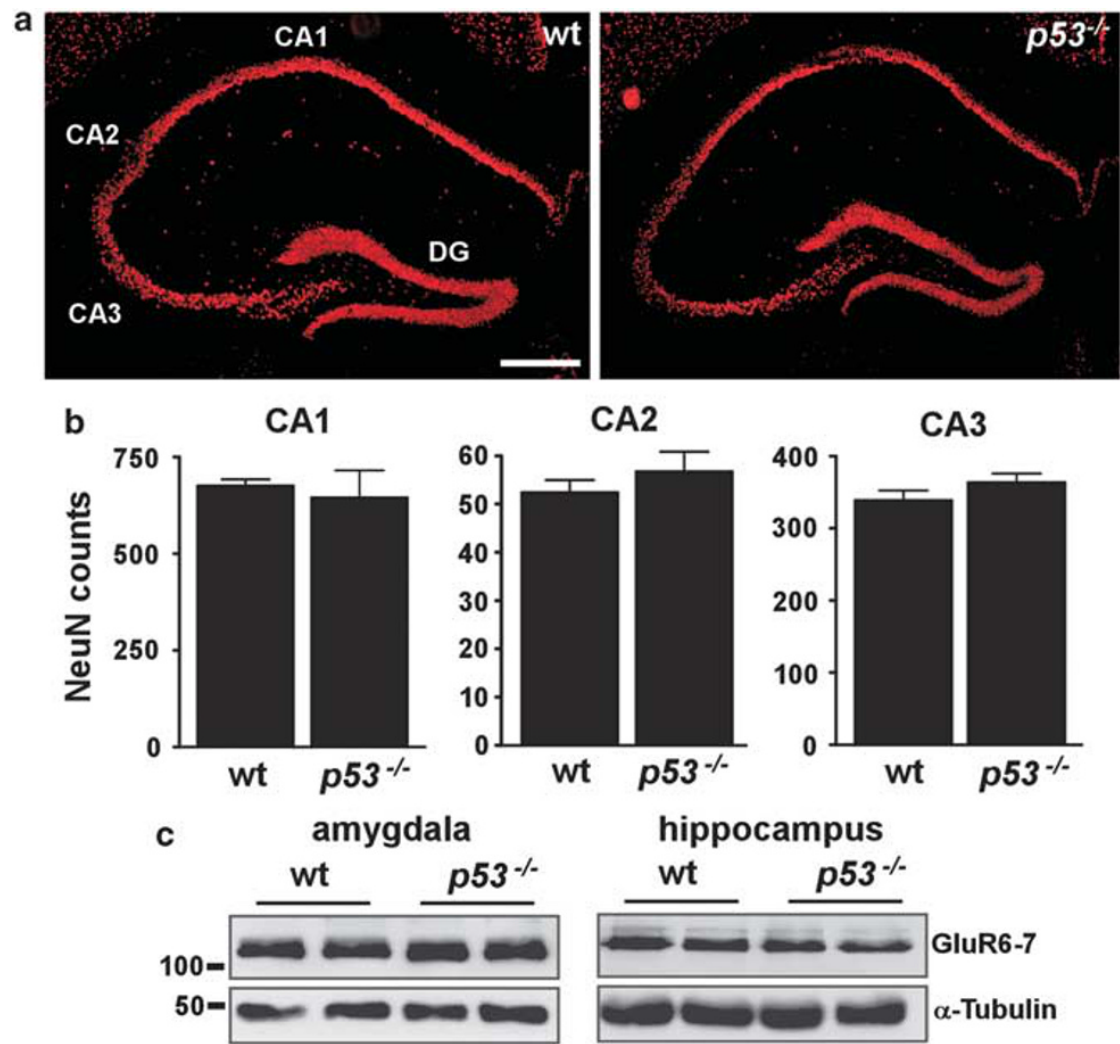

hippocampus

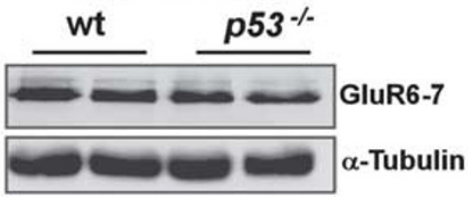

Figure 1 Normal brain architecture in p53-deficient mice. (a) Representative NeuN-stained sections from naïve wild-type (wt) and p53-deficient (p53 ${ }^{-1-}$ ) brains at the level of dorsal hippocampus showing normal-appearing organization of neuronal populations. CA, cornu ammonis; DG, dentate gyrus. Scale bar, $400 \mu$ m. (b) Graphs showing average neuron counts from NeuN-stained sections of dorsal hippocampus from naïve brains for three major subfields. No significant differences were found for any subfield ( $n=3$ per group). (c) Representative western blots ( $n=1$ per lane) showing equivalent levels of the KA receptor GluR6-7 in the amygdala and hippocampus of each genotype. Molecular weight markers in kilodaltons are depicted to the left. $\alpha$-tubulin is included below as a guide to protein loading 

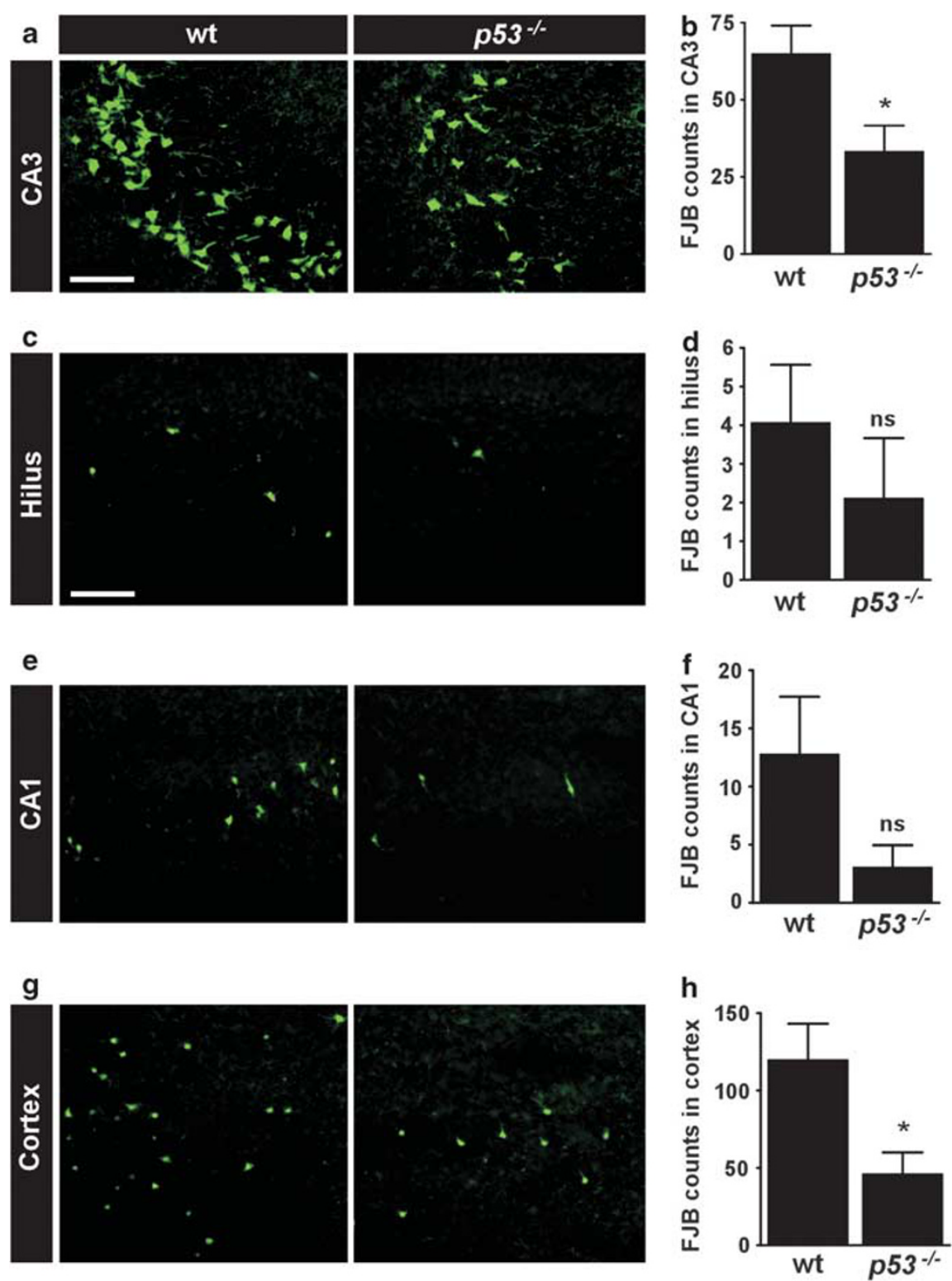

Figure 2 Region-specific acute neuroprotection in p53-deficient mice after status epilepticus. (a-h) Representative photomicrographs showing FJB staining of degenerating neurons in various ipsilateral fields and corresponding graphs quantifying counts for wild-type (wt) and p53-deficient ( $p 53^{-1-}$ ) mice $72 \mathrm{~h}$ after status epilepticus. Note the significantly reduced neuronal death in $p 53^{-1-}$ mice in ( $\mathbf{a}$ and $\left.\mathbf{b}\right) \mathrm{CA} 3$ and $(\mathbf{g}$ and $\mathbf{h}$ ) neocortex. Lens in $a, x 40$; lens in $c, e, g, x 20$. Scale bar in $a, 75 \mu \mathrm{m} ; c$ (for $c, e, g$ ) $150 \mu \mathrm{m}$. Data are from $n=10-20$ per group. ${ }^{*} P<0.05$ compared to wt. $n s$, non-significant

Bid, which are both under p53 control, ${ }^{27,28}$ as well as the seizure-regulated $\mathrm{BH} 3$-only protein $\mathrm{Bim},{ }^{29}$ in wild-type and p53-deficient mice after status epilepticus. Bid, Bax and Bim were not differently expressed in p53-deficient mice compared with wild-type mice after status epilepticus (see Supplementary Figure, S1).

Prolonged electrographic seizures during status epilepticus in p53-deficient mice. Previous studies of status epilepticus in p53-deficient mice did not undertake EEG analysis. ${ }^{12,13}$ To establish whether or not p53-deficient mice underwent an episode of status epilepticus equivalent to wild-type mice, we equipped animals from both groups with skull-mounted electrodes and recorded and quantified EEG between the time of KA microinjection and administration of lorazepam to curtail seizures. Unexpectedly, the sum duration of high amplitude and high frequency electrographic seizures was significantly longer in p53-deficient mice compared with wild-type mice (Figure 3a and b).

Exacerbated epileptic phenotype in p53-deficient mice. We next sought to characterize the emergence and phenotype of epilepsy in p53-deficient mice. For this we used 
a

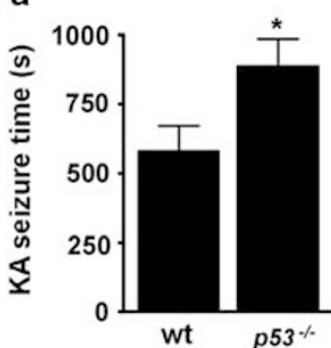

b

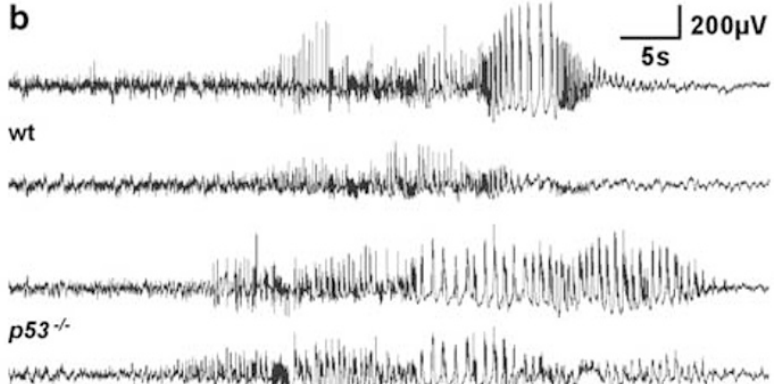

Figure 3 Increased electrographic seizures after KA in p53-deficient mice. (a) Graph showing sum of high amplitude and high frequency seizure time during status epilepticus in wild-type (wt) and p53-deficient $\left(p 53^{-/-}\right)$mice $\left(n=8-12\right.$ per group). ${ }^{*} P<0.05$ compared with wild-type. (b) Representative traces of electrographic seizures recorded after KA using surface electrodes in mice of each genotype. Note the extended polyspiking in $p 53^{-/-}$mice

a

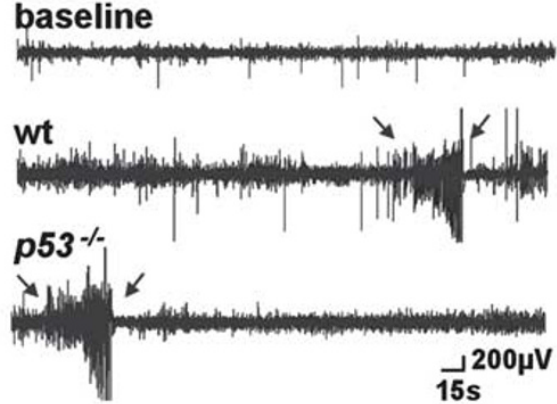

C

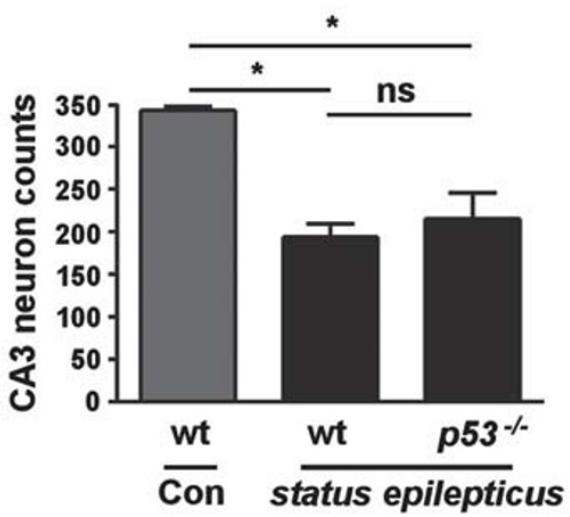

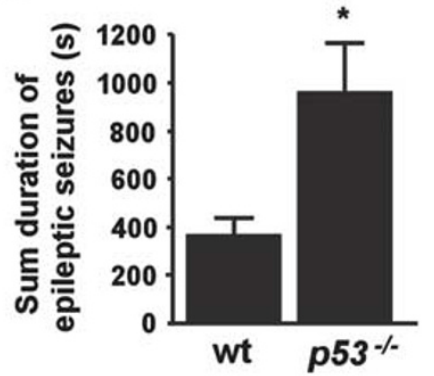

d
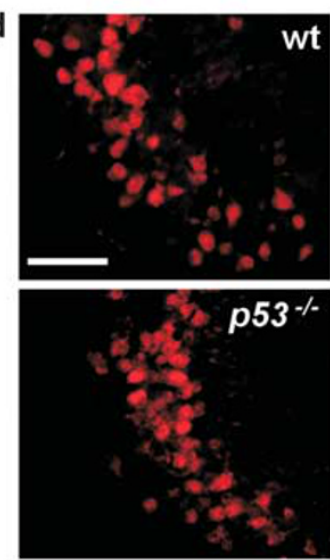

Figure 4 Increased spontaneous seizures and dissipation of hippocampal neuroprotection in p53-deficient mice 2 weeks after status epilepticus. (a) Representative EEG telemetry traces showing captured spontaneous seizures (spiking between arrows) in wild-type (wt) and p53-deficient (p53-1-) mice during epilepsy monitoring. (b) Graph showing that the sum duration of spontaneous seizures in $p 53^{-/-}$mice was significantly longer than wt over the period of epilepsy monitoring. ${ }^{\star} P<0.05$ compared with wildtype ( $n=6$ per group). (c) Graph showing neuron counts in the ipsilateral CA3 subfield of mice 2 weeks after status epilepticus. Wt $(n=7)$ and $p 53^{-1-}(n=6)$ mice subject to status epilepticus displayed significantly reduced CA3 counts compared with control (Con) non-seizure wild-type mice $(n=2)$. CA3 counts between genotypes subject to status epilepticus were non-significant (ns). ${ }^{*} P<0.05$. (d) Representative NeuN-staining of the ipsilateral CA3 subfield (x40 lens) from both genotypes 2 weeks after status epilepticus. Note the similarity of neuronal survival within the CA3 subfields. Scale bar, $75 \mu \mathrm{m}$

continuous EEG monitoring via implanted telemetry units. Using this system we recently characterized the epileptic phenotype following intra-amygdala KA-induced status epilepticus in C57BL/6 mice. ${ }^{26}$ Mice exhibit spontaneous seizures 3 days following status epilepticus, with all mice developing epilepsy by day 5 . Mice typically display 1-4 spontaneous seizures per day. ${ }^{26}$ To investigate whether absence of p53 influences epilepsy in this model, we implanted wild-type and p53-deficient mice with EEG telemetry units, subjected them to status epilepticus as before, and then continuously recorded EEG beginning on day 3 until the end of the second week post-status epilepticus.

Representative EEG traces of spontaneous seizures from telemetry recordings are shown in Figure 4a. The emergent epileptic phenotype in wild-type mice was 
essentially the same as that previously reported in this model. ${ }^{26}$ That is, all mice developed epilepsy within the first week following status epilepticus and averaged $\sim 2$ spontaneous seizures per day. Individual spontaneous seizures lasted just under $20 \mathrm{~s}$ each. Monitoring of p53-deficient mice following status epilepticus determined that these mice also all developed epilepsy. Further, over the 2-week recordings, the sum duration of spontaneous seizures was significantly longer in p53-deficient compared with wild-type mice (Figure 4b). p53-deficient mice tended to have more frequent spontaneous seizures and the duration of a typical spontaneous seizure was $\sim 28 \%$ longer compared with wild-type mice. Details of these and other measured parameters are presented in the Supplementary Table (see Supplementary Figure S2). Mice which received intra-amygdala vehicle did not display any spontaneous seizures $(n=2$, data not shown).

\section{Equivalence of hippocampal damage between wild-type} and p53-deficient mice after long-term epilepsy monitoring. On completion of the 2 weeks epilepsy monitoring, brains from mice of both groups were processed to assess hippocampal damage. Both wild-type and p53-deficient mice subjected to status epilepticus had significantly fewer surviving ipsilateral CA3 pyramidal neurons compared with non-seizure vehicle-injected wildtype mice (Figure 4c). However, p53-deficient mice subjected to status epilepticus did not have significantly different numbers of surviving CA3 neurons than wild-type seizure mice (Figure $4 c$ and $d$ ).

Neuropeptide $Y$ re-organization in epileptic p53-deficient mice. Dentate gyrus rearrangement, including mossy fiber sprouting is a common pathologic feature of temporal lobe epilepsy. We next investigated changes to dentate gyrus organization by staining hippocampal sections from the epileptic mice for neuropeptide $\mathrm{Y}$, which is a marker of synaptic rearrangement.

Neuropeptide $Y$ staining in non-seizure control mice was mainly present in scattered hilar neurons and occasionally in cells surrounding the stratum pyramidale layer. Diffuse lowlevel staining was also evident in the outer molecular layer of the dentate gyrus (Figure $5 \mathrm{a}$ ). In contrast, neuropeptide $Y$ staining was markedly increased in the hippocampus of epileptic wild-type mice (Figure 5a). This was most evident in the stratum radiatum of the CA3a pyramidal neurons (data not shown) and in the outer molecular layer of the dentate gyrus (Figure $5 \mathrm{a}$ and b). Neuropeptide $\mathrm{Y}$ staining in epileptic

Figure 5 Neuropeptide $Y$ rearrangement in p53-deficient and wild-type mice following status epilepticus. (a) Representative double-staining of NeuN (red) and neuropeptide $Y$ (NPY, green) of the dentate gyrus region in non-seizure control wild-type mice ( $w t+$ veh), wild-type mice subject to status epilepticus ( $w t+\mathrm{SE}$ ) and p53-deficient mice subject to status epilepticus ( $\left.p 53^{-/-}+\mathrm{SE}\right)$. Note enhanced NPY staining (green) in both genotypes subject to status epilepticus. DG, dentate gyrus, mol, molecular layer. Scale bar, $300 \mu \mathrm{m}$. (b) Graph showing NPY scores for the dentate gyrus in sections taken following epilepsy monitoring. NPY was not significantly (ns) different between genotypes ( $n=5-6$ per group) p53-deficient mice followed a similar pattern and semiquantitative analysis found no significant difference compared with wild-type mice (Figure $5 a$ and $b$ ).
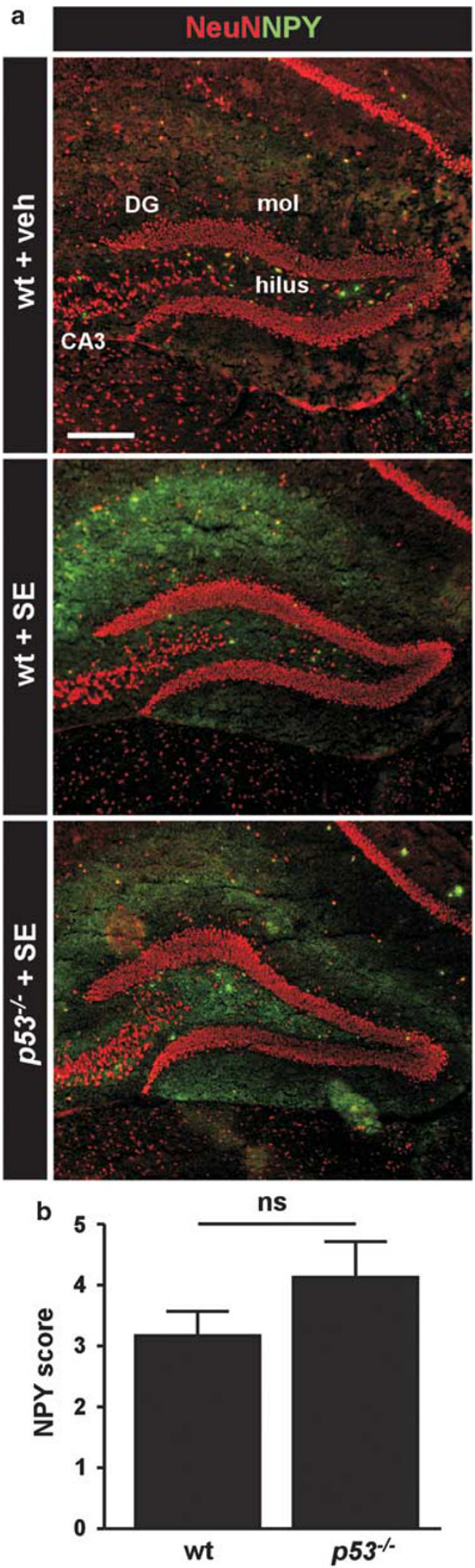


\section{Discussion}

Previous investigation of the role of p53 in epilepsy models focused on putatively pro-apoptotic effects on acute seizureinduced neuronal death. The present study confirmed that p53 regulates acute seizure-induced neuronal death in vivo, and also shows that loss of p53 is associated with prolonged electrographic status epilepticus and, chronically, development of a more severe epileptic phenotype in mice. Thus, we identify two novel consequences of p53 deficiency. This study challenges the suitability of broad targeting of p53 for neuroprotection and points to neurophysiologic and possibly post-insult roles for $\mathrm{p53}$, which may impact on the process of epileptogenesis.

The functional significance of p53 for acute seizure-induced neuronal death is largely established. Induction of p53 is commonly found in rodent brain following prolonged seizures, while blocking p53 pharmacologically or genetically, spares a significant population of hippocampal neurons from degeneration. ${ }^{9,10,12,14,30}$ In the present study, loss of p53 transiently protected certain hippocampal neurons from status epilepticus induced by intra-amygdala KA in mice, in accordance with our previous findings, ${ }^{8}$ and work by others. ${ }^{12,13}$ We found, as in other kainate models, ${ }^{12}$ that p53-deficient mice also display reduced cortical neuronal death after status epilepticus. Thus, p53 directly contributes to acute seizure-induced neuronal death in vivo. The molecular mechanism of the protection probably derives from inhibition of the $\mathrm{BH} 3-o n l y$ protein Puma. Indeed, expression of Puma is blocked in p53-deficient mice and by pifithrin- $\alpha$, and these mice are protected against status epilepticus. $^{8}$ In contrast, while Bim-deficient mice display hippocampal neuroprotection in the present model, ${ }^{29} \mathrm{Bim}$ was not differently expressed in p53-deficient mice. Likewise, we found that Bid, another p53-regulated $\mathrm{BH} 3$-only protein, ${ }^{28}$ was normally expressed in p53-deficient mice, and loss of Bid does not alter seizure-induced neuronal death in this model. ${ }^{31}$ Thus, Puma remains the most likely effector of p53-induced neuronal death after status epilepticus in this model. Whether p73, a homolog of p53, is important in the present model is unknown, but p73 functions have been proposed to overlap with p53 in regulating cell death. ${ }^{32}$

The present study identified a novel neurophysiologic phenotype resulting from the absence of p53. Mice lacking p53 develop a more severe electrographic status epilepticus. This contrasts with a previous study which, using behavioral scoring, had reported that p53 deficiency does not influence seizure severity. ${ }^{12}$ This discrepancy highlights the importance of using EEG as opposed to behaviour-only assessment to grade status epilepticus. The present study did not explore the cause of the phenotype. We did not find any obvious hippocampal or amygdala abnormalities in naïve p53-deficient mice, which could account for the observed effect, although differences may exist at a cell structure or molecular level. Indeed, p53 has been implicated in control of neurite branching, ${ }^{19}$ and a recent study identified a p53-dependent gene which modulated seizure susceptibility and $\mathrm{GABA}_{\mathrm{A}}$ ( $\gamma$-amino butyric acid) receptor expression. ${ }^{21}$ Also, although p53-deficient mice are born normally, ${ }^{33}$ loss of p53 has been associated with decreased expression of a mitochondrial enzyme, ${ }^{34}$ developmental abnormalities, ${ }^{25}$ brain tumors ${ }^{12}$ and exencephaly. ${ }^{24}$ It is also notable that mice lacking effective DNA repair, a function under p53 control, display earlier-onset KA-induced seizures. ${ }^{15}$ The presence of a proepileptic neurophysiologic phenotype in p53-deficient mice suggests it may be preferable to target genes under p53 control such as Puma rather than p53 itself to yield neuroprotection. ${ }^{8}$

Assessment of the longevity of hippocampal neuroprotection in p53-deficient mice has produced conflicting findings. Morrison et al. reported that protection against kainate persisted in p53-deficient mice for 1 week or longer, ${ }^{12}$ while Djebali et al. found that initial protective effects were absent by 7 days after status epilepticus. ${ }^{13}$ Here, we observed similarsized hippocampal lesions in p53-deficient mice to wild-type mice at the end of epilepsy monitoring. Thus, in our model, as with Djebali et al., hippocampal cell loss in p53-deficient mice progressed to match the wild-type lesion. Although Djebali et al. concluded delayed neuronal death must be p53-independent ${ }^{13}$ a secondary explanation is that the more severe status epileticus in p53-deficient mice was responsible. Notably, mice lacking the p53 target gene puma do not display protracted seizures after KA and these mice continue to display protection long after status epilepticus. ${ }^{8}$ It is also possible that spontaneous seizures in p53-deficient mice caused additional neuron loss thereby narrowing the difference with wild-type mice. However, spontaneous seizures probably do not cause progressive neuron loss in epileptic animals. ${ }^{35}$ Loss of other functions of p53 may be accountable for dissipation of neuroprotection over extended periods. p53 has roles in DNA repair, and effective DNA repair machinery is important for protecting against seizure-damage. ${ }^{15,36}$ Thus, our data suggest that while acute seizure-induced neuronal death comprises a p53-regulated component, the delayed neuronal death in our model may be p53-independent or arise from extended status epilepticus.

The present study also found that p53-deficient mice develop a more severe epileptic phenotype following status epilepticus. This was unexpected. Indeed, other pharmacologic or genetic neuroprotective manipulations using the present model have translated to a reduction in the occurrence of spontaneous seizures. ${ }^{8,18}$ If unrelated to a declining influence of the early neuroprotection, the exacerbated epileptic phenotype in p53-deficient mice may be the result of extended status epilepticus in these animals. Indeed, in an opposite scenario where the duration of status epilepticus was reduced, animals developed fewer spontaneous seizures. ${ }^{37}$ Alternatively, loss of certain p53 functions may have direct pro-epileptic consequences. Which p53-regulated genes are responsible of course is unknown. Post-injury axon outgrowth and regeneration may be altered in p53-deficient mice, ${ }^{20}$ and we may speculate that p53 contributes to adaptive repair processes, which subvert epileptic seizures. Deregulation of genes under p53 control which have been implicated in inhibitory neurotransmission ${ }^{21}$ might also be responsible. Regardless, the p53-dependent process(es) disrupted presumably do not impact on gross hippocampal changes which, at least when assayed by neuropeptide $Y$ rearrangement, were not different between p53-deficient and wild-type animals. Levels of p53 are elevated in the epileptic focus of patients with temporal lobe 
epilepsy, ${ }^{11}$ therefore, rather than being interpreted as a sign of ongoing cell death, higher p53 levels might serve to influence ictogenesis or other aspects of the epileptogenic process in humans.

One caveat of the present study is that an influence of genetic background cannot be entirely ruled out. Indeed, when p53-deficient mice were crossed to a non-C57BL/6 background they were no longer found to be protected against systemic $\mathrm{KA}^{38}$ In our model, however, damage variance between genetic backgrounds is quite limited, ${ }^{39}$ particularly compared with the systemic KA model used in the earlier report. $^{38}$

In summary, the present study revealed that, loss of p53 results in a neurophysiologic phenotype and exacerbated the emergent epileptic phenotype following status epilepticus. Given that p53 levels are altered in chronic human epilepsy, this may indicate a potentially antiepileptic role for p53 beyond its pro-apoptotic effects acutely. Wholesale targeting of p53 may be unsuitable for protection against status epilepticus, but identification, among the repertoire of genes under p53 control, of the cause of this phenotype may lead to potentially novel antiepileptic or antiepileptogenic therapeutic targets.

\section{Materials and Methods}

Epilepsy model. All animal procedures were performed in accordance with the principals of the European Communities Council Directive (86/609/EEC) and were approved by the Research Ethics Committee of the Royal College of Surgeons in Ireland. Adult (20-25 g) male mice were used and were obtained from Jackson Laboratory, Bar Harbor, ME, USA ( $p 53^{+/+,-/-}$on a C57BL/6 background). Genotyping of mice was performed using DNA extracted from tail snips in accordance with the supplier's recommendations for Trp53 ${ }^{\text {tm1Tyj }}$ (Jackson Laboratory). Food and water were available ad libitum. Status epilepticus was induced by unilateral stereotaxic microinjection of KA into the amygdala, as described. ${ }^{8}$ Briefly, mice were first anesthetized with isoflurane (3-5\% induction, $1-2 \%$ maintenance) and kept normothermic in a stereotaxic frame. Next, mice were affixed with skull-mounted electrodes (Bilaney Consultants Ltd., Sevenoaks, UK) above the dorsal hippocampus and the frontal cortex to record surface EEG using a Grass Comet digital EEG (Medivent Ltd., Lucan, Ireland). A guide cannula was affixed over the dura (coordinates from Bregma: $A P=-0.94 ; \mathrm{L}=-2.85 \mathrm{~mm}$ ) and the entire skull assembly fixed in place with dental cement. Anesthesia was discontinued, EEG recordings were commenced, and then a 31-gauge internal cannula was inserted into the lumen of the guide to inject KA (Ocean Produce International, Nova Scotia, Canada) $(0.3 \mu \mathrm{g}$ in $0.2 \mu \mathrm{l}$ vehicle; phosphate-buffered saline (PBS), pH adjusted to 7.4) into the amygdala. Non-seizure control mice received $0.2 \mu \mathrm{l}$ intra-amygdala vehicle. Lorazepam $(6 \mathrm{mg} / \mathrm{kg}$, intraperitoneal) was administered 40 min after KA, and the EEG was recorded for up to $1 \mathrm{~h}$ thereafter. Mice were euthanized at different time points after anticonvulsant and brains flash-frozen whole in 2-methylbutane at $-30^{\circ} \mathrm{C}$. Brains from additional naive (non-instrumented) wild-type and p53-deficient mice were used to examine hippocampal and amygdala neuroanatomy and gene expression.

Epilepsy monitoring. Long-term EEG recordings to define the emergence of epilepsy was performed using implantable EEG telemetry units (Data Systems International, St. Paul, MN, USA), as described. ${ }^{8,18,26}$ Transmitters (Model: F20-EET, DSI) were implanted in a subcutaneous pocket and used to record bilateral EEG from skull overlying the dorsal ipsilateral and contralateral hippocampi. EEG data were acquired using the Dataquest A.R.T. system. Following surgery, mice received intraamygdala KA to elicit status epilepticus and then telemetry units were activated from day 3 until day 14. After recordings, mice were euthanized and brains processed for histopathology as described below. Telemetry EEG recordings were manually analyzed for occurrence of epileptic seizures. These were defined as high frequency $(>5 \mathrm{~Hz})$ high amplitude ( $>2 \times$ baseline) polyspike discharges of $\geq 5 \mathrm{~s}$ duration.

Western blotting. Western blotting was performed as previously described. ${ }^{8}$ For analysis of naïve wild-type and p53-deficient mice, both hippocampi or bilateral amygdala tissue punches were obtained. For analysis of $\mathrm{Bcl}-2$ family proteins after seizures in wild-type and p53-deficient mice, ipsilateral hippocampus was obtained $8 \mathrm{~h}$ after lorazepam injection. Tissue was homogenized in a lysis buffer, proteins boiled in gel-loading buffer, separated on SDS-PAGE gels and transferred onto nitrocellulose membranes. Membranes were then incubated overnight with primary antibodies against $\alpha$-tubulin (Santa Cruz Biotechnology, Heidelberg, Germany), Bid (R \& D Systems Europe Ltd, Abingdon, UK), Bim (Enzo Life Sciences (UK) Ltd., Exeter, UK) and GluR6/7 (1:1000, Chemicon, Hampshire, UK). Membranes were then incubated with horseradish peroxidase-conjugated secondary antibodies (Jackson ImmunoResearch, Plymouth, PA, USA) and bands visualized using Supersignal West Pico Chemiluminescent Substrate (Pierce, Rockford, IL, USA). Images were captured using a Fuji-film LAS-300 (Fuji, Sheffield, UK) and densitometry performed using AlphaEaseFC4.0 gel-scanning integrated optical density software (Alpha Innotech, San Leandro, CA, USA).

Histopathology. Histopathology was undertaken as described. ${ }^{8,26}$ For assessment of acute neuronal damage, fresh-frozen brain sections at the level of the dorsal hippocampus were air-dried, post-fixed in formalin and processed for FJB staining (Millipore, Cork, Ireland). For immunohistochemistry, sections were first blocked in goat serum and then incubated overnight with NeuN (Chemicon) or neuropeptide Y (Sigma-Aldrich Ireland, Dublin, Ireland). Sections were then washed and incubated with goat anti-mouse or goat anti-rabbit secondary antibodies conjugated to AlexaFluor 488 or 568 (Bio Sciences Ltd., Dun Laoghaire, Ireland). Sections were mounted and imaged using a Nikon $2000 \mathrm{~s}$ epifluorescence microscope with a Hamamatsu Orca 285 camera (Micron-optica, Enniscorthy, Ireland). Semi-quantification of damaged and/or surviving cells was performed on the side ipsilateral to $\mathrm{KA}$ injection for the entire $\mathrm{CA} 3$ subfield, beginning at the border with CA2 through to the end of CA3C/CA4, as well as the hilus, CA1 and neocortex. Counts were the average of two adjacent sections assessed by an observer blinded to experimental group/condition. Neuropeptide $Y$ staining was used as a marker of hippocampal re-organization. Staining was assessed on a semi-quantitative rating scale according to previously reported criteria. ${ }^{40}$ Briefly, Score 0 , no neuropeptide $Y$ between the tips and crest of the dentate gyrus; Score 1, sparse staining in the supragranular region in a patchy distribution between the tips and crest of the dentate gyrus; Score 2, more abundant neuropeptide $Y$ in the supragranular region in a continuous distribution between tips and crest of the dentate gyrus; Score 3, prominent neuropeptide $Y$ in the supragranular region in a continuous pattern between tips and crest, with occasional patches of confluent staining between tips and crest of the dentate gyrus; Score 4, prominent neuropeptide $Y$ in the supragranular region that form a confluent dense laminar band between tips and crest and Score 5, confluent dense laminar band of neuropeptide $Y$ in the supragranular region that extends into the inner molecular layer.

Data analysis. Data are presented as mean \pm S.E.M. Data were analyzed using analysis of variance with post hoc Fisher's PLSD test or for two group comparison Student's t-test (StatView software; SAS Institute, Inc., Cary, NC, USA). Significance was accepted at $P<0.05$.

\section{Conflict of interest}

The authors declare no conflict of interest.

Acknowledgements. The authors would like to thank Nikolaus Plesnila for helpful discussion. This work was supported by grants from Health Research Board Ireland (RP/2005/24, RP/2007/37), Science Foundation Ireland (04/IN3/B466, 08/IN1/B1875, 08/RFP/NSC1745) and fellowships (to TE) from the Irish Research Council for Science Engineering and Technology and Health Research Board.

1. Pitkanen A. Drug-mediated neuroprotection and anti-epileptogenesis. Neurology 2002; 59(Suppl. 5): S27-S33.

2. Acharya MM, Hattiangady B, Shetty AK. Progress in neuroprotective strategies for preventing epilepsy. Prog Neurobiol 2008; 84: 363-404

3. Engel T, Henshall DC. Apoptosis, Bcl-2 family proteins and caspases: the ABCs of seizure-damage and epileptogenesis? Int J Physiol Pathophysiol Pharmacol 2009; 1 : $97-115$.

4. Morrison RS, Kinoshita Y. The role of p53 in neuronal cell death. Cell Death Differ 2000; 7 868-879 
5. Xiang H, Hochman DW, Saya H, Fujiwara T, Schwartzkroin PA, Morrison RS. Evidence for p53-mediated modulation of neuronal viability. J Neurosci 1996; 16: 6753-6765.

6. Jordan J, Galindo MF, Prehn JH, Weichselbaum RR, Beckett M, Ghadge GD et al. p53 expression induces apoptosis in hippocampal pyramidal neuron cultures. J Neurosci 1997; 17: $1397-1405$.

7. Cregan SP, Arbour NA, Maclaurin JG, Callaghan SM, Fortin A, Cheung EC et al. p53 activation domain 1 is essential for PUMA upregulation and p53-mediated neuronal cell death. J Neurosci 2004; 24: 10003-10012.

8. Engel T, Murphy BM, Hatazaki S, Jimenez-Mateos EM, Concannon CG, Woods I et al. Reduced hippocampal damage and epileptic seizures after status epilepticus in mice lacking proapoptotic Puma. FASEB J 2010; 24: 853-861.

9. Sakhi S, Sun N, Wing LL, Mehta P, Schreiber SS. Nuclear accumulation of p53 protein following kainic acid-induced seizures. Neuroreport 1996; 7: 493-496.

10. Liu W, Rong Y, Baudry M, Schreiber SS. Status epilepticus induces p53 sequence-specific DNA binding in mature rat brain. Brain Res Mol Brain Res 1999; 63: 248-253.

11. Engel T, Murphy BM, Schindler CK, Henshall DC. Elevated p53 and lower MDM2 expression in hippocampus from patients with intractable temporal lobe epilepsy. Epilepsy Res 2007; 77: 151-156.

12. Morrison RS, Wenzel HJ, Kinoshita Y, Robbins CA, Donehower LA, Schwartzkroin PA Loss of the p53 tumor suppressor gene protects neurons from kainate- induced cell death. J Neurosci 1996; 16: 1337-1345

13. Djebaili M, Lerner-Natoli M, Pascale M, Baille V, Bockaert J, Rondouin G. Molecular events involved in neuronal death induced in the mouse hippocampus by in-vivo injection of kainic acid. Brain Res Mol Brain Res 2001; 93: 190-198.

14. Culmsee C, Zhu X, Yu QS, Chan SL, Camandola S, Guo Z et al. A synthetic inhibitor of p53 protects neurons against death induced by ischemic and excitotoxic insults, and amyloid beta-peptide. J Neurochem 2001; 77: 220-228.

15. Neema M, Navarro-Quiroga I, Chechlacz M, Gilliams-Francis K, Liu J, Lamonica K et al. DNA damage and nonhomologous end joining in excitotoxicity: neuroprotective role of DNA-PKcs in kainic acid-induced seizures. Hippocampus 2005; 15: 1057-1071.

16. Ebert U, Brandt $C$, Loscher W. Delayed sclerosis, neuroprotection, and limbic epileptogenesis after status epilepticus in the rat. Epilepsia 2002; 43(Suppl 5): 86-95.

17. Brandt $\mathrm{C}$, Potschka H, Loscher W, Ebert U. N-methyl-D-aspartate receptor blockade after status epilepticus protects against limbic brain damage but not against epilepsy in kainate model of temporal lobe epilepsy. Neuroscience 2003; 118: 727-740.

18. Jimenez-Mateos EM, Hatazaki S, Johnson MB, Bellver-Estelles C, Mouri G, Bonner C et al. Hippocampal transcriptome after status epilepticus in mice rendered seizure damagetolerant by epileptic preconditioning features suppressed calcium and neuronal excitability pathways. Neurobiol Dis 2008; 32: 442-453.

19. Di Giovanni S, Knights CD, Rao M, Yakovlev A, Beers J, Catania J et al. The tumor suppressor protein p53 is required for neurite outgrowth and axon regeneration. EMBO 2006; 25: 4084-4096.

20. Tedeschi A, Di Giovanni S. The non-apoptotic role of p53 in neuronal biology: enlightening the dark side of the moon. EMBO Rep 2009; 10: 576-583.

21. Cheung CC, Yang C, Berger T, Zaugg K, Reilly $P$, Elia AJ et al. Identification of BERP (brain-expressed RING finger protein) as a p53 target gene that modulates seizure susceptibility through interacting with GABA(A) receptors. Proc Natl Acad Sci USA 2010; 107: 11883-11888.

22. Amson R, Lassalle JM, Halley H, Prieur S, Lethrosne F, Roperch JP et al. Behaviora alterations associated with apoptosis and down-regulation of presenilin 1 in the brains of p53-deficient mice. Proc Natl Acad Sci USA 2000; 97: 5346-5350.

23. Maeda K, Hata R, Gillardon F, Hossmann K-A. Aggravation of brain injury after transient focal ischemia in p53-deficient mice. Mol Brain Res 2001; 88: 54-61.

24. Sah VP, Attardi LD, Mulligan GJ, Williams BO, Bronson RT, Jacks T. A subset of p53-deficient embryos exhibit exencephaly. Nat Genet 1995; 10: 175-180.

25. Armstrong JF, Kaufman MH, Harrison DJ, Clarke AR. High-frequency developmental abnormalities in p53-deficient mice. Curr Biol 1995; 5: 931-936.

26. Mouri G, Jimenez-Mateos E, Engel T, Dunleavy M, Hatazaki S, Paucard A et al. Unilateral hippocampal CA3-predominant damage and short latency epileptogenesis after intra-amygdala microinjection of kainic acid in mice. Brain Res 2008; 1213 140-151.

27. Miyashita T, Krajewski S, Krajewska M, Wang HG, Lin HK, Liebermann DA et al. Tumor suppressor p53 is a regulator of bcl-2 and bax gene expression in vitro and in vivo. Oncogene 1994; 9: 1799-1805.

28. Sax JK, Fei P, Murphy ME, Bernhard E, Korsmeyer SJ, EI-Deiry WS. BID regulation by p53 contributes to chemosensitivity. Nat Cell Biol 2002; 4: 842-849.

29. Murphy BM, Engel T, Paucard A, Hatazaki S, Mouri G, Tanaka K et al. Contrasting patterns of Bim induction and neuroprotection in Bim-deficient mice between hippocampus and neocortex after status epilepticus. Cell Death Differ 2010; 17: 459-468.

30. Sakhi S, Bruce A, Sun N, Tocco G, Baudry M, Schreiber SS. p53 induction is associated with neuronal damage in the central nervous system. Proc Natl Acad Sci USA 1994; 91 $7525-7529$.

31. Engel T, Caballero-Caballero A, Schindler CK, Plesnila N, Strasser A, Prehn JH et al. The $\mathrm{BH} 3-$ only protein Bid is dispensable for seizure-induced neuronal death and the associated nuclear accumulation of apoptosis-inducing factor (AIF). J Neurochem 2010; 115: 92-101.

32. Melino G, De Laurenzi V, Vousden KH. p73: Friend or foe in tumorigenesis. Nat Rev Cancer 2002; 2: 605-615.

33. Donehower LA, Harvey M, Slagle BL, McArthur MJ, Montgomery Jr CA, Butel JS et al. Mice deficient for p53 are developmentally normal but susceptible to spontaneous tumours. Nature 1992; 356: 215-221.

34. Araki N, Morimasa T, Sakai T, Tokuoh H, Yunoue S, Kamo M et al. Comparative analysis of brain proteins from p53-deficient mice by two-dimensional electrophoresis. Electrophoresis 2000; 21: 1880-1889.

35. Gorter JA, Goncalves Pereira PM, van Vliet EA, Aronica E, Lopes da Silva FH Lucassen PJ. Neuronal cell death in a rat model for mesial temporal lobe epilepsy is induced by the initial status epilepticus and not by later repeated spontaneous seizures. Epilepsia 2003; 44: 647-658.

36. Quach N, Chan T, Lu TA, Schreiber SS, Tan Z. Induction of DNA repair proteins, Ref-1 and XRCC1, in adult rat brain following kainic acid-induced seizures. Brain Res 2005; 1042 236-240.

37. Pitkanen A, Kharatishvili I, Narkilahti S, Lukasiuk K, Nissinen J. Administration of diazepam during status epilepticus reduces development and severity of epilepsy in rat. Epilepsy Res 2005; 63: 27-42.

38. Schauwecker PE, Steward O. Genetic determinants of susceptibility to excitotoxic cell death: implications for gene targeting approaches. Proc Natl Acad Sci USA 1997; 94 4103-4108.

39. Tanaka K, Jimenez-Mateos EM, Matsushima S, Taki W, Henshall DC. Hippocampa damage after intra-amygdala kainic acid-induced status epilepticus and seizure preconditioning-mediated neuroprotection in SJL mice. Epilepsy Res 2010; 88: 151-161.

40. Cavazos JE, Golarai G, Sutula TP. Mossy fiber synaptic reorganization induced by kindling: time course of development, progression, and permanence. $J$ Neurosci 1991; 11 2795-2803.

(c)

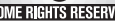

Cell Death and Disease is an open-access journal published by Nature Publishing Group. This work is licensed under the Creative Commons Attribution-Noncommercial-No Derivative Works 3.0 Unported License. To view a copy of this license, visit http://creativecommons.org/licenses/by-nc-nd/3.0/

Supplementary Information accompanies the paper on Cell Death and Disease website (http://www.nature.com/cddis) 